\title{
Simultaneous state and fault estimation for Takagi-Sugeno implicit models with Lipschitz constraints
}

\author{
Manal Ouzaz, Abdellatif El Assoudi, Jalal Soulami* and El Hassane El Yaagoubi \\ Laboratory of High Energy Physics and Condensed Matter, Faculty of Science Hassan II University of \\ Casablanca, B.P 5366, Maarif, Casablanca, Morocco \\ ECPI, Department of Electrical Engineering, ENSEM Hassan II University of Casablanca, B.P 8118, Oasis, \\ Casablanca Morocco \\ manal.ouzaz@gmail.com,a.elassoudi@ensem.ac.ma,jalal.soulami@gmail.com, h.elyaagoubi@ensem.ac.ma
}

\section{ARTICLE INFO}

\section{Article History:}

Received 15 October 2019

Accepted 10 May 2020

Available 03 January 2021

Keywords:

Takagi-Sugeno implicit model

Estimation of actuator

and sensor faults

Fuzzy Observer design

Lyapunov method

LMI technique

Lipschitz constraints

AMS Classification 2010:

93C95; 93C42; $93 C 10$

93005; 68740

\author{
ABSTRACT
}

This paper presents a state and fault observer design for a class of TakagiSugeno implicit models (TSIMs) with unmeasurable premise variables satisfying the Lipschitz constraints. The fault variable is constituted by the actuator and sensor faults. The actuator fault affects the state and the sensor fault affects the output of the system. The approach is based on the separation between dynamic and static relations in the TSIM. Firstly, the method begins by decomposing the dynamic equations of the algebraic equations. Secondly, the fuzzy observer design that satisfies the Lipschitz conditions and permits to estimate simultaneously the unknown states, actuator and sensor faults is developed. The aim of this approach for the observer design is to construct an augmented model where the fault variable is added to the state vector. The exponential convergence of the state estimation error is studied by using the Lyapunov theory and the stability condition is given in term of only one linear matrix inequality (LMI). Finally, numerical simulation results are given to highlight the performances of the proposed method by using a TSIM of a single-link flexible joint robot.

\section{Introduction}

Due to the growth of the industrial demand for high reliability and safety, the field of the observer design for many chemical and physical processes by using different approaches has attracted much attention of the researchers for a long time. This is due to its wide and successful use in the areas of control, fault detection and diagnosis (FDD) and fault tolerant control (FTC). We may cite e.g. [1-6] and the references therein.

In few decades, fuzzy control systems based on the Takagi-Sugeno (T-S) approach has been received great attention in researches and recognized as a powerful tool to describe the global behaviors of nonlinear systems. The interest of this formalism is to apprehend the global behavior of a nonlinear process by a set of local linear models, [7, 8]. Once the T-S fuzzy models are obtained, some linear control methodologies can be used see e.g. [9, 10]. Many research works on observation and control of T-S models have been discussed in literature $11-13$.

Likewise, due to the fact that many industrial systems are modeled by implicit models which are also called singular models or descriptor models, several researches have been done on implicit systems. Implicit systems are applied to many applications such as power systems, economic systems, electrical systems, chemical systems and so on, see e.g. 14 17. Such systems are used to describe a larger class of systems than the normal

*Corresponding author 
system model and to design controller and observer in different areas of research. Notice that, in [18, 19, a fuzzy implicit model is defined by extending the ordinary T-S fuzzy model [7].

In this paper, based on the T-S approach, we study the problem of observer design for simultaneous state and fault estimation for a class of continuous-time TSIMs in presence of actuator and sensor faults. In the literature, many works on fuzzy observer design and its application to FDD and FTC for T-S fuzzy models exist in both explicit and implicit cases see e.g. [20 27] for T-S explicit models case and 28 37] for T-S implicit models. It should be noted that, generally an interesting way to solve the various observer problems raised previously is to write the convergence conditions on the LMI form 38.

The main contribution of this paper is to develop a new methodology of simultaneous state and fault estimation for a class of TSIMs with unmeasurable premise variables satisfying the Lipschitz constraints. Based on the separation between the dynamic and static relations in the TSIM, the augmented system constituted of dynamic equations and fault vector is constructed. The exponential stability of the augmented state estimation error is studied by using the Lyapunov theory and the stability condition is given in term of only one LMI. Besides, the proposed fuzzy observer is synthesized by only an explicit structure.

The rest of this paper is organized as follows: Section 2 presents the class of TSIMs including actuator and sensor faults. In section 3, the fuzzy observer is designed allowing the simultaneous state and fault estimation. Finally, Section 4 applies this result on the TSIM of the single-link flexible joint robot which allows a clear evaluation of the performance of the proposed method.

The next notations are useful:

- $A^{T}$ represents the transposed matrix of $A$.

- $A>0$ indicates that $A$ is a matrix symmetric and positive definite.

- $I$ and 0 indicate respectively the identity matrix and the zero matrix with the appropriate dimension.

- $\mathbf{R}^{n}$ and $\mathbf{R}^{n \times m}$ denote the spaces of $n$ dimensional real vectors and $n \times m$ real matrices, respectively.

\section{Takagi-Sugeno implicit model description}

The following class of T-S fuzzy implicit models with unmeasurable premise variables in presence of actuator and sensor faults is considered:

$$
\left\{\begin{aligned}
M \dot{z} & =\sum_{i=1}^{q} \varphi_{i}(z)\left(A_{i} z+B_{i} u+F_{a i} f_{a}\right) \\
y & =C z+D u+D_{a} f_{a}+F_{s} f_{s}
\end{aligned}\right.
$$

where $z=\left[\begin{array}{ll}Z_{1}^{T} & Z_{2}^{T}\end{array}\right]^{T} \in \mathbf{R}^{n}$ is the state vector with $Z_{1} \in \mathbf{R}^{r}$ is the vector of differential variables, $Z_{2} \in \mathbf{R}^{n-r}$ is the vector of algebraic variables, $u \in \mathbf{R}^{m}$ is the control input, $y \in \mathbf{R}^{p}$ is the measured output vector. $q$ is the number of submodels. $f_{a} \in \mathbf{R}^{n_{a}}$ and $f_{s} \in \mathbf{R}^{n_{s}}$ are the actuator fault and sensor fault, respectively. $A_{i} \in \mathbf{R}^{n \times n}$, $B_{i} \in \mathbf{R}^{n \times m}, C \in \mathbf{R}^{p \times n}, D \in \mathbf{R}^{p \times m}, D_{a} \in \mathbf{R}^{p \times n_{a}}$, $F_{a i} \in \mathbf{R}^{n \times n_{a}}, F_{s} \in \mathbf{R}^{p \times n_{s}}$ and $M \in \mathbf{R}^{n \times n}$ are real known constant matrices with:

$$
\left\{\begin{array}{c}
A_{i}=\left(\begin{array}{cc}
A_{i}^{11} & A_{i}^{12} \\
A_{i}^{21} & A_{i}^{22}
\end{array}\right) ; \quad B=\left(\begin{array}{c}
B_{i}^{1} \\
B_{i}^{2}
\end{array}\right) \\
F_{a i}=\left(\begin{array}{c}
F_{a i}^{1} \\
F_{a i}^{2}
\end{array}\right) ; C=\left(\begin{array}{ll}
C^{1} & 0
\end{array}\right)
\end{array}\right.
$$

where $A_{i}^{22}$ are supposed invertible. $M$ is assumed to be of the form:

$$
M=\left(\begin{array}{ll}
I & 0 \\
0 & 0
\end{array}\right)
$$

and satisfies $\operatorname{rank}(M)=r<n$. Notice that if $M$ is not of the form (3), without loss of generality, we can always find two nonsingular matrices $X \in \mathbb{R}^{n \times n}$ and $Y \in \mathbb{R}^{n \times n}$ such that the following relation is satisfied [17]:

$$
X M Y=\left(\begin{array}{ll}
I & 0 \\
0 & 0
\end{array}\right)
$$

$\varphi_{i}(z)$ are the weighting functions that ensure the transition between the contribution of each sub model:

$$
\left\{\begin{array}{cl}
M \dot{z} & =A_{i} z+B_{i} u+F_{a i} f_{a} \\
y & =C z+D u+D_{a} f_{a}+F_{s} f_{s}
\end{array}\right.
$$

They verify the so-called convex sum property:

$$
\sum_{i=1}^{q} \varphi_{i}(z)=1 ; \quad 0 \leq \varphi_{i}(z) \leq 1
$$

Assumption 1. Suppose that [17]:

- $\left(M, A_{i}\right)$ is regular $\left(\operatorname{det}\left(s M-A_{i}\right) \neq 0 \forall s \in \mathbf{C}\right)$.

- All sub-models (5) are impulse observable and detectable.

As mentioned above, our approach consists to separate the differential equations from the algebraic equations in each sub-model (5) and the global 
fuzzy model is obtained by aggregation of the resulting sub-models. Indeed, from (2) and (3), submodel (5) can be rewritten as follows:

$$
\left\{\begin{array}{c}
\dot{Z}_{1}=A_{i}^{11} Z_{1}+A_{i}^{12} Z_{2}+B_{i}^{1} u+F_{a i}^{1} f_{a} \\
0=A_{i}^{21} Z_{1}+A_{i}^{22} Z_{2}+B_{i}^{2} u+F_{a i}^{2} f_{a} \\
y=C^{1} Z_{1}+D u+D_{a} f_{a}+F_{s} f_{s}
\end{array}\right.
$$

which is equivalent to the following form:

$$
\left\{\begin{array}{c}
\dot{Z}_{1}=M_{i} Z_{1}+N_{i} u+P_{a i} f_{a} \\
Z_{2}=J_{i} Z_{1}+K_{i} u+L_{a i} f_{a} \\
y=C^{1} Z_{1}+D u+D_{a} f_{a}+F_{s} f_{s}
\end{array}\right.
$$

where

$$
\left\{\begin{aligned}
M_{i} & =A_{i}^{11}+A_{i}^{12} J_{i} \\
N_{i} & =B_{i}^{1}+A_{i}^{12} K_{i} \\
P_{a i} & =F_{a i}^{1}+A_{i}^{12} L_{a i} \\
J_{i} & =-\left(A_{i}^{22}\right)^{-1} A_{i}^{21} \\
K_{i} & =-\left(A_{i}^{22}\right)^{-1} B_{i}^{2} \\
L_{a i} & =-\left(A_{i}^{22}\right)^{-1} F_{a i}^{2}
\end{aligned}\right.
$$

Let

$$
f=\left(\begin{array}{c}
f_{a} \\
f_{s}
\end{array}\right)
$$

So, system (8) can be rewritten under the equivalent state representation given by:

$$
\left\{\begin{array}{c}
\dot{Z}_{1}=M_{i} Z_{1}+N_{i} u+P_{i} f \\
Z_{2}=J_{i} Z_{1}+K_{i} u+L_{i} f \\
y=C^{1} Z_{1}+D u+T f
\end{array}\right.
$$

where

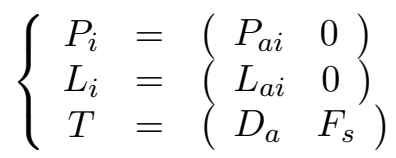

So, from (11) it follows that:

$$
\varphi_{i}(z)=\varphi_{i}\left(Z_{1}, Z_{2}\right)=\varphi_{i}(\lambda)
$$

with $\lambda=\left[\begin{array}{lll}Z_{1}^{T} & u^{T} & f^{T}\end{array}\right]^{T}$.

Then, the following equivalent form of TSIM (1) can be obtained:

$$
\left\{\begin{aligned}
\dot{Z}_{1} & =\sum_{i=1}^{q} \varphi_{i}(\lambda)\left(M_{i} Z_{1}+N_{i} u+P_{i} f\right) \\
Z_{2} & =\sum_{i=1}^{q} \varphi_{i}(\lambda)\left(J_{i} Z_{1}+K_{i} u+L_{i} f\right) \\
y & =C^{1} Z_{1}+D u+T f
\end{aligned}\right.
$$

Assumption 2. : Suppose that the fault $f$ is of the following form:

$$
f=\alpha_{0}+\alpha_{1} t+\ldots+\alpha_{n_{f}} t^{n_{f}}
$$

where the $\left(n_{f}+1\right)^{t h}$ time derivative of $f$ is null (i.e. $f^{\left(n_{f}+1\right)}=0$ ) and $\alpha_{j}, j=0,1, \ldots, n_{f}$ are unknown constant parameters.

Let

$$
\eta_{j}=f^{(j-1)} j=1, \ldots, n_{f}+1
$$

Then, we have:

$$
\left\{\begin{array}{ccc}
\dot{\eta}_{1} & = & \eta_{2} \\
\dot{\eta}_{2} & = & \eta_{3} \\
& \vdots & \\
\dot{\eta}_{n_{f}} & = & \eta_{n_{f}+1} \\
\dot{\eta}_{n_{f}+1} & = & 0
\end{array}\right.
$$

Thus, the equivalent augmented form of system (14) can be written:

$$
\left\{\begin{aligned}
\dot{\xi}_{1} & =\sum_{i=1}^{q} \varphi_{i}(\mu)\left(\Phi_{i} \xi_{1}+\Psi_{i} u\right) \\
\xi_{2} & =\sum_{i=1}^{q} \varphi_{i}(\mu)\left(\Omega_{i} \xi_{1}+K_{i} u\right) \\
y & =R \xi_{1}+D u
\end{aligned}\right.
$$

where

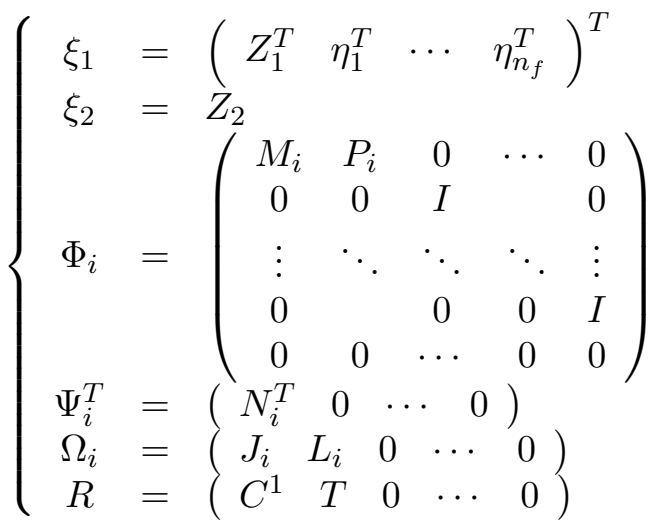

and

$$
\mu=\left[\begin{array}{ll}
\xi_{1}^{T} & u^{T}
\end{array}\right]^{T}
$$

In order to design an observer for system (1), we introduce the following matrices:

$$
\left\{\begin{array}{l}
\Phi_{0}=\frac{1}{q} \sum_{i=1}^{q} \Phi_{i} \\
\bar{\Phi}_{i}=\Phi_{i}-\Phi_{0} \\
\Psi_{0}=\frac{1}{q} \sum_{i=1}^{q} \Psi_{i} \\
\bar{\Psi}_{i}=\Psi_{i}-\Psi_{0}
\end{array}\right.
$$

By using 21, system (18) takes the following equivalent form:

$$
\left\{\begin{aligned}
\dot{\xi}_{1}= & \Phi_{0} \xi_{1}+\Psi_{0} u \\
& +\sum_{i=1}^{q} \varphi_{i}(\mu)\left(\bar{\Phi}_{i} \xi_{1}+\bar{\Psi}_{i} u\right) \\
\xi_{2}= & \sum_{i=1}^{q} \varphi_{i}(\mu)\left(\Omega_{i} \xi_{1}+K_{i} u\right) \\
y= & R \xi_{1}+D u
\end{aligned}\right.
$$

\section{Main result}

Based on the form (22), the proposed fuzzy observer design of TSIM (1) takes the following 
structure:

$$
\left\{\begin{aligned}
\dot{\hat{\xi}}_{1}= & \Phi_{0} \hat{\xi}_{1}+\Psi_{0} u-H(\hat{y}-y) \\
& +\sum_{i=1}^{q} \varphi_{i}(\hat{\mu})\left(\bar{\Phi}_{i} \hat{\xi}_{1}+\bar{\Psi}_{i} u\right) \\
\hat{\xi}_{2}= & \sum_{i=1}^{q} \varphi_{i}(\hat{\mu})\left(\Omega_{i} \hat{\xi}_{1}+K_{i} u\right) \\
\hat{y}= & R \hat{\xi}_{1}+D u
\end{aligned}\right.
$$

where $\left(\hat{\xi}_{1}, \hat{\xi}_{2}\right), \hat{y}$ and $\hat{\mu}$ denote the estimate of $\left(\xi_{1}, \xi_{2}\right), y$ and $\eta$ respectively. Matrix $H$ is to be determined such that $\left(\hat{\xi}_{1}, \hat{\xi}_{2}\right)$ converges toward $\left(\xi_{1}, \xi_{2}\right)$ exponentially.

In order to establish the asymptotic convergence condition of the observer (23), we define:

$$
\varepsilon=\left(\begin{array}{c}
\varepsilon_{1} \\
\varepsilon_{2}
\end{array}\right)=\left(\begin{array}{c}
\hat{\xi}_{1}-\xi_{1} \\
\hat{\xi}_{2}-\xi_{2}
\end{array}\right)
$$

From systems 22 and 23 , the estimation error dynamics is given by:

$$
\left\{\begin{aligned}
\dot{\varepsilon}_{1}= & \Gamma_{0} \varepsilon_{1}+\Delta \\
\varepsilon_{2}= & \sum_{i=1}^{q}\left(\varphi_{i}(\hat{\mu})-\varphi_{i}(\mu)\right)\left(\Omega_{i} \xi_{1}+K_{i} u\right) \\
& +\sum_{i=1}^{q} \varphi_{i}(\hat{\mu}) \Omega_{i} \varepsilon_{1}
\end{aligned}\right.
$$

where

$$
\Gamma_{0}=\Phi_{0}-H R
$$

and

$$
\Delta=\sum_{i=1}^{q}\left(\bar{\Phi}_{i} \Delta_{1}+\Delta_{2} u\right)
$$

with

$$
\left\{\begin{array}{l}
\Delta_{1}=\varphi_{i}(\hat{\mu}) \hat{\xi}_{1}-\varphi_{i}(\mu) \xi_{1} \\
\Delta_{2}=\bar{\Psi}_{i}\left(\varphi_{i}(\hat{\mu})-\varphi_{i}(\mu)\right)
\end{array}\right.
$$

Assumption 3. : Assume that the following conditions hold:

$$
\left\{\begin{aligned}
\left|\Delta_{1}\right| & <\delta_{i}\left|\varepsilon_{1}\right| \\
\left|\Delta_{2}\right| & <\beta_{i}\left|\varepsilon_{1}\right| \\
|u| & <\gamma
\end{aligned}\right.
$$

where $\delta_{i}, \beta_{i}$ are positives scalars Lipschitz constants and $\gamma>0$.

Let

$$
\theta=\sum_{i=1}^{q}\left(\sigma\left(\bar{\Phi}_{i}\right) \delta_{i}+\beta_{i} \gamma\right)
$$

where $\sigma\left(\bar{\Phi}_{i}\right)$ denotes the maximum singular value of the matrix $\bar{\Phi}_{i}$.

Then, by using Assumption 3 , the term $\Delta$ can be bounded as follows:

$$
|\Delta|<\theta\left|\varepsilon_{1}\right|
$$

The following result can be stated.
Theorem 1.: Under above Assumption 3, the system (25) is globally exponentially stable if given $\rho>0$ there exists matrices $P>0, Q>0$ and $W$ verifying the following $L M I$ :

$$
\left(\begin{array}{cc}
\Sigma+\theta^{2} Q+2 \rho P & P \\
P & -Q
\end{array}\right)<0
$$

where

$$
\Sigma=\Phi_{0}^{T} P-R^{T} W^{T}+P \Phi_{0}-W R
$$

The gain $H$ of the observer (23) is computed by:

$$
H=P^{-1} W
$$

Proof of Theorem 11: Notice that to prove the global asymptotic stability toward zero of the system (25), it suffices to prove that $\varepsilon_{1}$ converges toward zero. Indeed, the following Lyapunov function is considered:

$$
V=\varepsilon_{1}^{T} P \varepsilon_{1} \quad P>0
$$

From (25), it follows that:

$$
\dot{V}=\varepsilon_{1}^{T}\left(\Gamma_{0}^{T} P+P \Gamma_{0}\right) \varepsilon_{1}+\Delta^{T} P \varepsilon_{1}+\varepsilon_{1}^{T} P \Delta
$$

Lemma 1. : For any matrices $X$ and $Y$ with appropriate dimensions, the following property holds for any invertible matrix $J$ :

$$
X^{T} Y+Y^{T} X \leq X^{T} J^{-1} X+Y^{T} J Y
$$

For $Q>0$, by applying Lemma 1 and Assumption 3. (36) becomes:

$$
\dot{V}<\varepsilon_{1}^{T}\left(\Gamma_{0}^{T} P+P \Gamma_{0}+P Q^{-1} P\right) \varepsilon_{1}+\Delta^{T} Q \Delta
$$

Taking into account (31), 38 becomes:

$$
\dot{V}<\varepsilon_{1}^{T}\left(\Gamma_{0}^{T} P+P \Gamma_{0}+P Q^{-1} P+\theta^{2} Q\right) \varepsilon_{1}
$$

Let $\rho>0$, to ensure the exponential convergence of the estimation error, the following condition must be guaranteed (see [39] as cited in [9]):

$$
\dot{V}<\varepsilon_{1}^{T}\left(\Gamma_{0}^{T} P+P \Gamma_{0}+P Q^{-1} P+\theta^{2} Q\right) \varepsilon_{1}
$$

That leads to the following condition:

$$
\Gamma_{0}^{T} P+P \Gamma_{0}+P Q^{-1} P+\theta^{2} Q+2 \rho P<0
$$

Then from (26), we can establish the LMI condition (32) of Theorem 1 by using the Schur complement and the following change of variables:

$$
W=P H
$$

Thus, from the Lyapunov stability theory, if the LMI condition (32) is satisfied, the system (25) 
is exponentially asymptotically stable. This completes the proof of Theorem 1 .

\section{Numerical illustration}

In this section, the proposed fuzzy observer design (23) is applied to a single-link flexible joint robot in order to estimate on-line the unknown states and the faults of actuator and sensor simultaneously. The TSIM that we consider here is given in 36] which is supposed to be affected by an actuator and sensor faults as follows:

$$
\left\{\begin{aligned}
M \dot{z} & =\sum_{i=1}^{2} \varphi_{i}(z)\left(A_{i} z+B u+F_{a} f_{a}\right) \\
y & =C z+F_{s} f_{s}
\end{aligned}\right.
$$

where $z=\left(z_{1}, z_{2}, z_{3}, z_{4}, z_{5}, z_{6}\right)^{T}$ is the state vector with $z_{1}$ and $z_{2}$ are the angles of rotations of the motor and the link respectively, $z_{3}$ and $z_{4}$ are their angular velocities, $z_{5}$ and $z_{6}$ are their angular accelerations. $\mathrm{u}$ is the control variable, $y$ is the output measurement vector, $f_{a}$ is the actuator fault and $f_{s}$ is the sensor fault.

$$
\begin{aligned}
& A_{1}=\left(\begin{array}{cccccc}
0 & 0 & 1 & 0 & 0 & 0 \\
0 & 0 & 0 & 1 & 0 & 0 \\
0 & 0 & 0 & 0 & 1 & 0 \\
0 & 0 & 0 & 0 & 0 & 1 \\
\frac{-k_{1}}{J_{m}} & \frac{k_{1}}{J_{m}} & \frac{-\beta}{J_{m}} & 0 & -1 & 0 \\
\frac{J_{L}}{J_{L}} & \nu_{\max } & 0 & 0 & 0 & -1
\end{array}\right) \\
& A_{2}=\left(\begin{array}{cccccc}
0 & 0 & 1 & 0 & 0 & 0 \\
0 & 0 & 0 & 1 & 0 & 0 \\
0 & 0 & 0 & 0 & 1 & 0 \\
0 & 0 & 0 & 0 & 0 & 1 \\
\frac{-k_{1}}{J_{m}} & \frac{k_{1}}{J_{m}} & \frac{-\beta}{J_{m}} & 0 & -1 & 0 \\
\frac{k_{1}}{J_{L}} & \nu_{\min } & 0 & 0 & 0 & -1
\end{array}\right) \\
& B^{T}=\left(\begin{array}{cccccc}
0 & 0 & 0 & 0 & \frac{k_{2}}{J_{m}} & 0
\end{array}\right) \\
& C=\left(\begin{array}{cccccc}
1 & 0 & 0 & 0 & 0 & 0 \\
0 & 0 & 1 & 0 & 0 & 0
\end{array}\right) \\
& M=\left(\begin{array}{ll}
I & 0 \\
0 & 0
\end{array}\right) \text { with } \operatorname{rank}(M)=4 \\
& F_{a}=B ; F_{s}=\left(\begin{array}{c}
0 \\
1
\end{array}\right)
\end{aligned}
$$

The weighting functions are given by:

$$
\left\{\begin{array}{l}
\varphi_{1}(z)=\frac{\nu-\nu_{\min }}{\nu_{\max }-\nu_{\min }} \\
\varphi_{2}(z)=\frac{\nu_{\max }-\nu}{\nu_{\max }-\nu_{\min }}
\end{array}\right.
$$

where $\nu$ is the premise variable having the expression below:

$$
\nu=-\frac{k_{1}}{J_{L}}-\frac{m g b}{J_{L}} \frac{\sin \left(z_{2}\right)}{z_{2}} \in\left[\nu_{\min }, \nu_{\max }\right]
$$

The expression of control variable and values of physical parameters are given in [36 and under assumption 2, the expression of unknown fault signals $f_{a}$ and $f_{s}$ are defined as in Figure 4 .

Therefore, to apply the proposed fuzzy observer (23) for the one-link flexible joint robot as stated in Theorem 1, it suffices to rewrite the model (43) into its equivalent form $(22)$ as mentioned above. Thus, by Theorem 1 with $\rho=2.5$ the following observer gain $H$ is obtained:

$$
H=\left(\begin{array}{rr}
37.5 & -4.1 \\
-25.0 & -60.2 \\
472.3 & -37.5 \\
246.9 & 55.6 \\
488.9 & 151.7 \\
-453.1 & 83.4 \\
1617.8 & 675.8 \\
-1993.7 & 463.2 \\
1986.6 & 977.5 \\
-3001.7 & 850.7
\end{array}\right)
$$

Simulation results with initial conditions:

$$
\begin{aligned}
& \xi_{1}(0)=\left[\begin{array}{lllllllllll}
0 & 0.1047 & 0 & 0 & 0 & 0 & 0 & 0 & 0 & 0
\end{array}\right]^{T} \\
& \hat{\xi}_{1}(0)=\left[\begin{array}{lllllllllll}
0 & 0.1147 & 0 & 0.001 & 0 & 0 & 0 & 0 & 0 & 0
\end{array}\right]^{T} \\
& \xi_{2}(0)=\left[\begin{array}{ll}
6.1756 & -5.6158
\end{array}\right]^{T} \\
& \hat{\xi}_{2}(0)=\left[\begin{array}{ll}
6.6620 & -6.1507
\end{array}\right]^{T}
\end{aligned}
$$

are given in Figure 1 to Figure 6 for which faults $f_{a}$ and $f_{s}$ are applied during the time intervals $[5,40]$ and $[50,85]$ respectively.

These simulation results show the performances of the proposed fuzzy observer (23) with the gain $H$ where the dashed lines denote the state variables, unknown faults and their derivatives estimated by the observer. They show that the observer gives a good estimation of unmeasurable states $z_{2}, z_{4}$, $z_{5}, z_{6}$, and unknown faults $f_{a}, f_{s}$ and their derivatives $\dot{f}_{a}, \dot{f}_{s}, \ddot{f}_{a}, \ddot{f}_{s}$ of the considered flexible robot. 

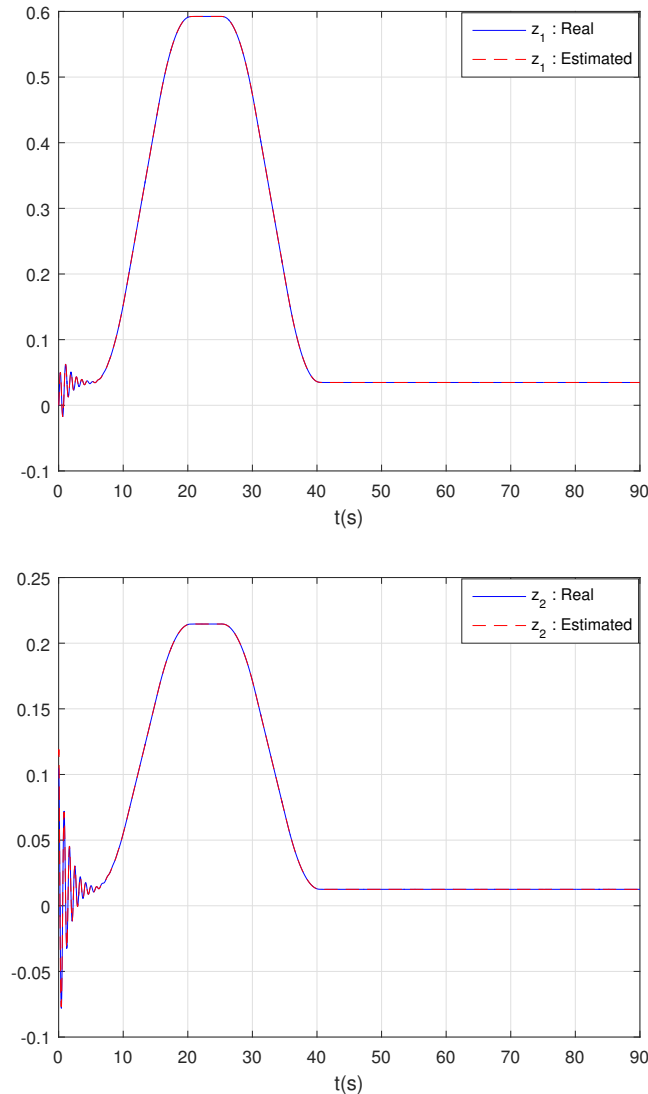

Figure 1. $z_{1}$ and $z_{2}$ with their estimates.
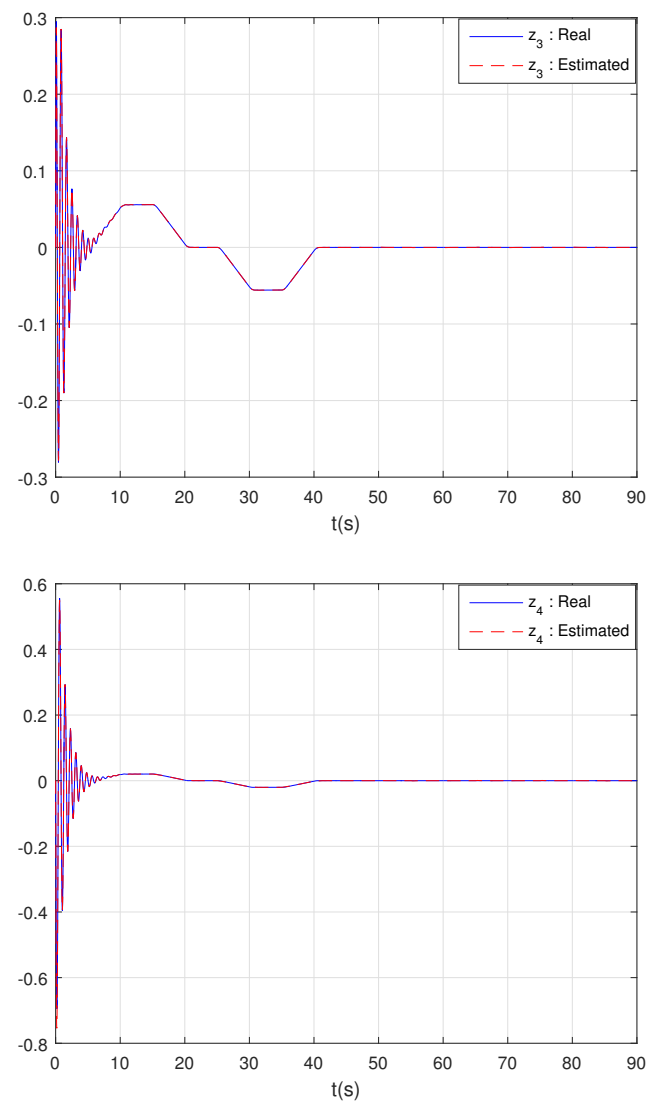

Figure 2. $z_{3}$ and $z_{4}$ with their estimates.
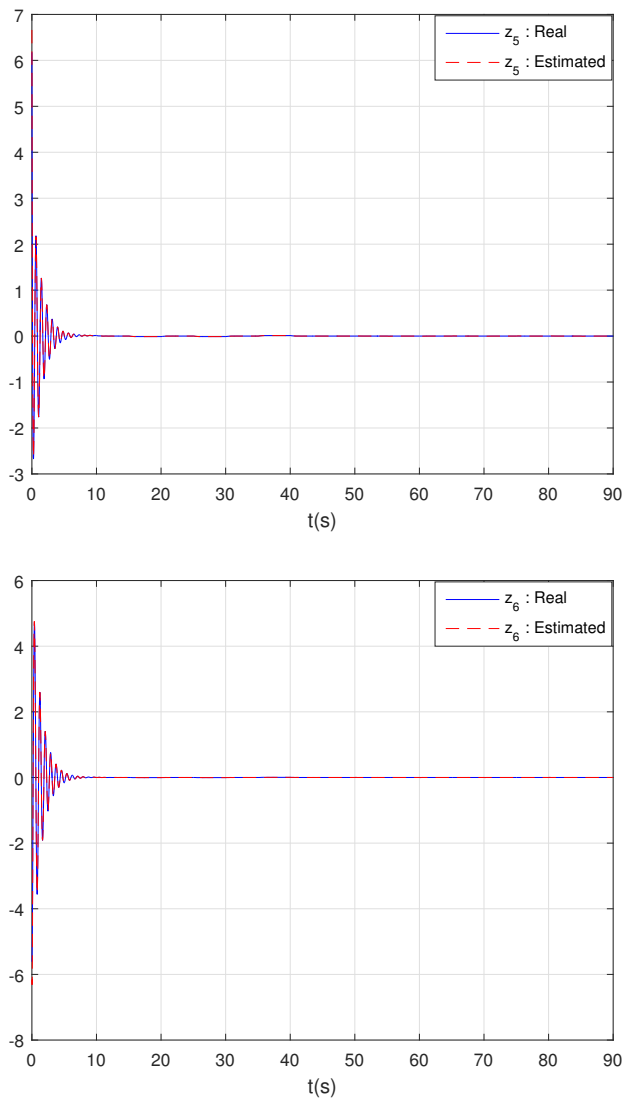

Figure 3. $z_{5}$ and $z_{6}$ with their estimates.
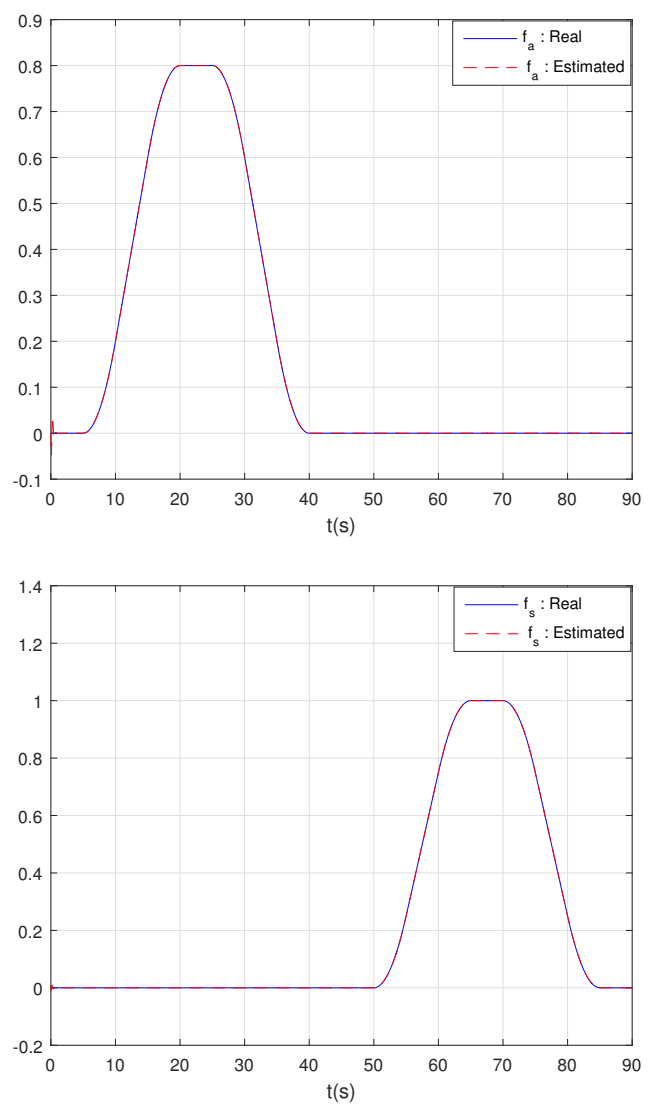

Figure 4. $f_{a}$ and $f_{s}$ with their estimates. 

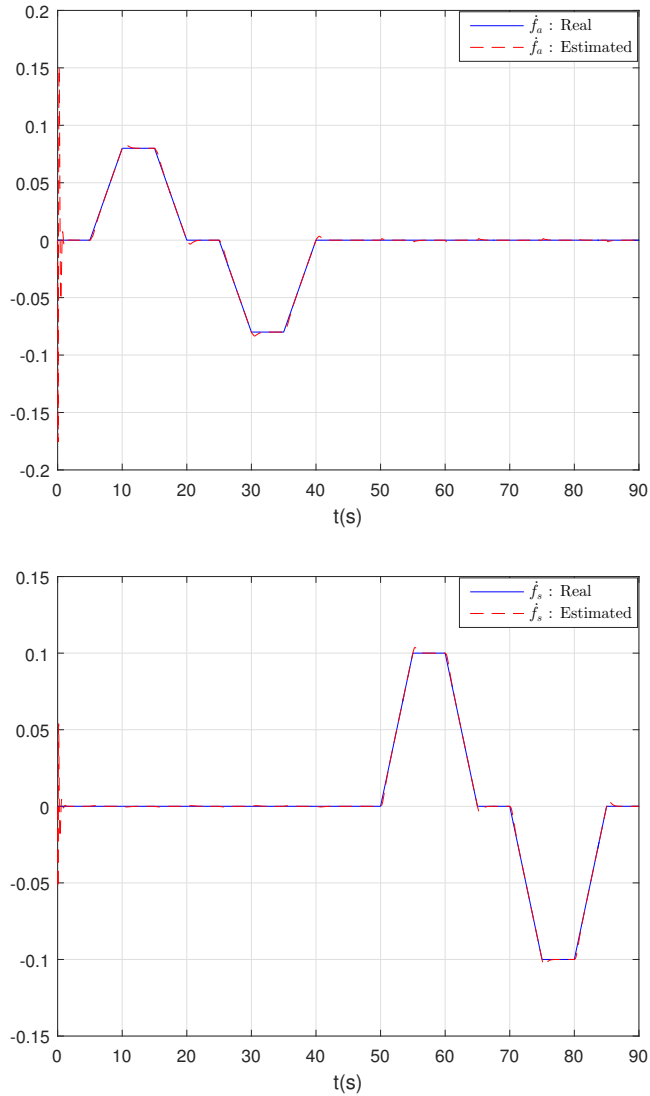

Figure 5. $\dot{f}_{a}$ and $\dot{f}_{s}$ with their estimates.
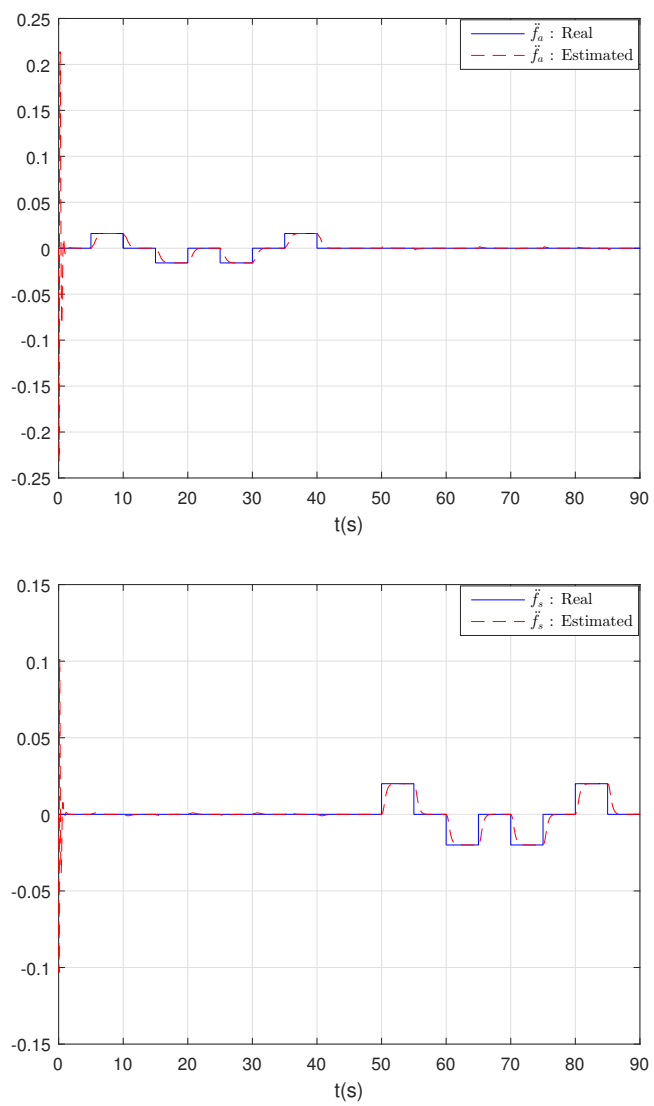

Figure 6. $\ddot{f}_{a}$ and $\ddot{f}_{s}$ with their estimates.

\section{Conclusion}

This paper develops a new methodology of fuzzy observer design, as a new contribution to simultaneously estimate fault variables as well as system states variables, for a class of TSIMs with unmeasurable premise variables satisfying the Lipschitz constraints in presence of the actuator and sensor faults. The approach is based on the separation between dynamic and static relations in the considered TSIM. The convergence of the state estimation error is studied by using the Lyapunov theory and the exponential stability conditions are given in term of only one LMI. In order to prove the performance of this observer design, a TSIM of the one-link flexible joint robot is studied. Simulation results are given and illustrated the effectiveness of the proposed approach.

\section{References}

[1] Isermann, R. (2006). Fault-Diagnosis Systems An Introduction from Fault Detection to Fault Tolerance. Springer-Verlag Berlin Heidelberg.

[2] Ding, S. X. (2008). Model-Based Fault Diagnosis Techniques: Design Schemes, Algorithms, and Tools. Berlin, Germany: Springer-Verlag.

[3] Lendek,Z., Guerra, T. M., Babuška, R., \& De Schutter, B.(2010). Stability Analysis and Nonlinear Observer Design Using TakagiSugeno Fuzzy Models. Springer-Verlag Berlin Heidelberg .

[4] Witczak, M. (2014).Fault Diagnosis and Fault-Tolerant Control Strategies for NonLinear Systems. Analytical and Soft Computing Approaches. Springer International Publishing Switzerland.

[5] Blanke, M., Kinnaert, M., Lunze, J., \& Staroswiecki, M.(2016).Diagnosis, and FaultTolerant Control. Springer-Verlag Berlin Heidelberg.

[6] Li, L. (2016). Fault Detection and FaultTolerant Control for Nonlinear Systems. Springer Fachmedien Wiesbaden.

[7] Takagi, T., Sugeno, M.(1985). Fuzzy identification of systems and its application to modeling and control, IEEE Trans. Syst., Man and Cybernetics, 15(1), 116-132.

[8] Taniguchi, T., Tanaka, K., Ohtake, H. ,\& Wang, H.(2001). Model construction, rule reduction, and robust compensation for generalized form of Takagi-Sugeno fuzzy systems. IEEE Transactions on Fuzzy Systems, 9(4), 525-538. 
[9] Tanaka, K., \& Wang, H. O. (2001). Fuzzy control systems design and analysis: A Linear Matrix Inequality Approach. John Wiley \& Sons.

[10] Lendek, Zs., Guerra, T.M., Babuška, R. \& De Schutter B. (2011). Stability analysis and nonlinear observer design using TakagiSugeno fuzzy models, Springer Berlin Heidelberg.

[11] Ichalal, D. ,Marx, B., Mammar, S., Maquin, D., \& Ragot, J. (2018). How to cope with unmeasurable premise variables in Takagi-Sugeno observer design: Dynamic extension approach. Engineering Applications of Artificial Intelligence, 67, 430-435.

[12] Wang, L., \& Lam, H. (2019). Further Study on Observer Design for Continuous-Time Takagi-Sugeno Fuzzy Model With Unknown Premise Variables via Average Dwell Time. IEEE Transactions on Cybernetics, 1-6.

[13] Xie, W.-B., Li, H., Wang, Z.-H., \& Zhang, J. (2019). Observer-based Controller Design for A T-S Fuzzy System with Unknown Premise Variables. International Journal of Control, Automation and Systems, 17(4), 907-915.

[14] Dai, L. (1989).Singular Control Systems. Lecture Notes in Control and Information Sciences. Springer-Verlag Berlin.

[15] Kumar, A.,\& Daoutidis, P. (1999). Control of nonlinear differential algebraic equation systems. Chapman \& Hall CRC.

[16] Kunkel, P., \& Mehrmann, V.(2006). Differential-Algebraic Equations-Analysis and Numerical Solution. Textbooks in Mathematics. European Mathematical Society. Zurich, Schweiz.

[17] Duan, G. R. (2010). Analysis and Design of Descriptor Linear Systems. Springer-Verlag New York.

[18] Taniguchi, T., Tanaka, K., Yamafuji, K., \& Wang, H. 0. (1999). Fuzzy Descriptor Systems: Stability Analysis and Design via LMIs. Proceedings of the American Control Conference. San Diego, California , 1827-1831.

[19] Taniguchi, T. Tanaka, K. ,\& Wang, H. 0. (2000). Fuzzy Descriptor Systems and Nonlinear Model Following Control. IEEE Transactions on Fuzzy Systems, 8(4), 442-452.

[20] Akhenak, A., Chadli, M.,Ragot , J., \& Maquin,D.(2009). Design of observers for Takagi-Sugeno fuzzy models for Fault Detection and Isolation. 7th IFAC Symposium on Fault Detection, Supervision and Safety of Technical Processes, SAFEPROCESS, Barcelona, Spain.
[21] Bouattour, M., Chadli, M., Chaabane, M., \& El Hajjaji, A.(2011). Design of Robust Fault Detection Observer for Takagi-Sugeno Models Using the Descriptor Approach. International Journal of Control, Automation, and Systems, 9(5), 973-979.

[22] Ichalal, D., Marx, B., Ragot, J., \& Maquin, D.(2012). New fault tolerant control strategies for nonlinear Takagi-Sugeno systems. International Journal of Applied Mathematics and Computer Science, 22(1), 197-210.

[23] Ichalal, D., Marx, B., Ragot, J., \& Maquin, D.(2014). Fault detection, isolation and estimation for Takagi-Sugeno nonlinear systems. Journal of the Franklin Institute, 351(7), 3651-3676.

[24] Youssef, T., Chadli, M. Karimi, H.R. ,\& Wang, R.(2016). Actuator and sensor faults estimation based on proportional integral observer for TS fuzzy model. Journal of the Franklin Institute, 354(6), 2524-2542.

[25] Ichalal, D., Marx, B. Ragot, J., Mammar, S., \& Maquin, D. (2016). Sensor fault tolerant control of nonlinear Takagi-Sugeno systems: Application to vehicle lateral dynamics. Int. J. Robust Nonlinear Control, 26(7), 1376-1394.

[26] Hadi, A. S., Shaker, M. S., \& Jawad, Q. A. (2019). Estimation/decoupling approach for robust Takagi-Sugeno UIO-based fault reconstruction in nonlinear systems affected by a simultaneous time-varying actuator and sensor faults. International Journal of Systems Science, 50(13), 2473-2485.

[27] Shaker, M. S. (2019). Hybrid approach to design Takagi-Sugeno observer-based FTC for non-linear systems affected by simultaneous time-varying actuator and sensor faults. IET Control Theory \& Applications, 13(5), 632641.

[28] Marx, B., Koenig ,D., \& Ragot, J. (2007). Design of observers for Takagi-Sugeno descriptor systems with unknown inputs and application to fault diagnosis. IET Control Theory and Applications, 1(5), 1487-1495.

[29] Mechmech, C., Hamdi, H. Rodrigues, M. ,\& Benhadj Braiek, N. (2012). State and unknown inputs estimations for multi-models descriptor systems. American Journal of Computational and Applied Mathematics, 2(3), 86-93.

[30] Hamdi, H., Rodrigues, M., Mechmeche, C., Theilliol, D. \& BenHadj Braiek, N.(2012). 
Fault detection and isolation for linear parameter varying descriptor systems via proportional integral observer. International Journal of Adaptive Control and Signal Processing, 26(3), 224-240.

[31] Aguilera-González, A., Astorga-Zaragoza, C. M., Adam-Medina, M., Theilliol, D., ReyesReyes, J.,\& Garcia-Beltrán, C. D.(2013). Singular linear parameter-varying observer for composition estimation in a binary distillation column. IET Control Theory \& Applications, 7(3), 411-422.

[32] Hamdi, H., Rodrigues, M. Mechmech, Ch., \& Benhadj Braiek, N.(2013). Observer based Fault Tolerant Control for Takagi-Sugeno Nonlinear Descriptor systems. International Conference on Control, Engineering \& Information Technology (CEIT'13). Proceedings Engineering \& Technology, 1, 52-57.

[33] Lopez-Estrada,F. R., Ponsart, J.C., Didier Theilliol, Astorga-Zaragoza, C. M.,\& Aberkane, S.(2014). Fault Diagnosis Based on Robust Observer for Descriptor-LPV Systems with Unmeasurable Scheduling Functions. 19th IFAC World Congress. Cape Town, South Africa. August 24-29, 47(3), 1079-1084.

[34] Lopez-Estrada, F. R., Ponsart, J.C., Didier Theilliol, Astorga-Zaragoza, C. M., \& CamasAnzueto, J. L.(2015). Robust Sensor Fault Estimation For Descriptor-LPV Systems with Unmeasurable Gain Scheduling Functions : Application to an Anaerobic Bioreactor. Int. J. Appl. Math. Comput. Sci., 25(2), 233-244.

[35] Bouassem, K., Soulami, J., El Assoudi, A., \& El Yaagoubi, E.(2016). Unknown Input Observer Design for a Class of Takagi-Sugeno Descriptor Systems. Nonlinear Analysis and Differential Equations, 4(10), 477-492.

[36] Louzimi, A., El Assoudi, A., Soulami, J., \& El Yaagoubi, E.(2017). Unknown Input Observer Design for a Class of Nonlinear Descriptor Systems: A Takagi-Sugeno Approach with Lipschitz Constraints. Nonlinear Analysis and Differential Equations, 5(3), 99-116.
[37] Bouassem, K., Soulami, J., El Assoudi, A., \& El Yaagoubi, E.(2017). Fuzzy Observer Design for a Class of Takagi-Sugeno Descriptor Systems Subject to Unknown Inputs. Nonlinear Analysis and Differential Equations, 5(3), 117-134.

[38] Boyd, S., \& al.(1994). Linear Matrix Inequalities in Systems and Control Theory. Philadelphia, PA: SIAM.

[39] Ichikawa, A., \& al.(1993). Control Hand Book. Ohmu Publisher, Tokyo in Japanese.

Manal Ouzaz was born in Agadir, Morocco, in 1987. She received the engineering degree in Electrical Engineering from National High School of Electricity and Mechanics (ENSEM), Casablanca, Morocco. She is currently working toward the PhD. degree at Hassan II University. Her research interests include observer design, model-based fault detection, fuzzy control.

(D) https://orcid.org/0000-0002-0815-7198

Abdellatif El Assoudi is a professor at the department of Electrical Engineering in National High School of Electricity and Mechanics (ENSEM), in Hassan II University of Casablanca (Morocco). His research interests focus in Nonlinear implicit model, Nonlinear observer design, Unknown Input Observer Design, fault detection, Takagi-Sugeno fuzzy control.

(10) https://orcid.org/0000-0001-8932-2134

Jalal Soulami is a professor at the department of Electrical Engineering in National High School of Electricity and Mechanics (ENSEM), in Hassan II University of Casablanca (Morocco). His research interests include Nonlinear implicit model, Nonlinear observer design, Unknown Input Observer Design, fault detection, Takagi-Sugeno fuzzy control.

(1) https://orcid.org/0000-0002-2082-7537

El Hassane El Yaagoubi is a professor at the department of Electrical Engineering in National High School of Electricity and Mechanics (ENSEM), in Hassan II University of Casablanca (Morocco). His areas of interest include Nonlinear implicit model, Nonlinear observer design, Unknown Input Observer Design, fault detection, Takagi-Sugeno fuzzy control. (D) https://orcid.org/0000-0002-4314-5870

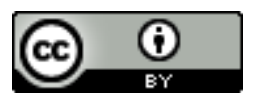

This work is licensed under a Creative Commons Attribution 4.0 International License. The authors retain ownership of the copyright for their article, but they allow anyone to download, reuse, reprint, modify, distribute, and/or copy articles in IJOCTA, so long as the original authors and source are credited. To see the complete license contents, please visit http://creativecommons.org/licenses/by/4.0/. 